\title{
Ultrasound-guided RNA interference targeting HIF-I alpha improves the effects of transarterial chemoembolization in rat liver tumors
}

This article was published in the following Dove Press journal:

OncoTargets and Therapy

27 November 2015

Number of times this article has been viewed

\author{
Cheng-Shi Chen ${ }^{1,2, *}$ \\ Qing Zhao',* \\ Sheng Qian' \\ Hai-Liang Li $^{2}$ \\ Chen-Yang Guo ${ }^{2}$ \\ Wei Zhang' \\ Zhi-Ping Yan' \\ Rong Liu' \\ Jian-Hua Wang'
}

'Department of Interventional

Radiology, Zhongshan Hospital, Fudan

University, Shanghai, People's Republic

of China; ${ }^{2}$ Department of Radiology,

Henan Cancer Hospital, Zhengzhou

University, Zhengzhou, People's

Republic of China

*These authors contributed equally to this work
Aim: To investigate whether ultrasound-guided RNA interference (RNAi) targeting hypoxiainducible factor-1alpha (HIF-1 $\alpha$ ) can enhance the efficacy of transarterial chemoembolization (TACE) in treating hepatocellular carcinoma.

Materials and methods: Rats with orthotopic hepatocellular carcinoma were randomized to four groups and treated as follows: 1) control; 2) siHIF-1 $\alpha$; 3) TACE; 4) siHIF-1 $\alpha+$ TACE. Lentivirus $\left(4 \times 10^{8}\right.$ transfection units) with or without small interfering RNA (siRNA) expression in $0.6 \mathrm{~mL}$ transduction reagent was injected into tumors using a standard $1 \mathrm{~mL}$ syringe under ultrasonic guidance. In the siHIF- $1 \alpha+$ TACE and siHIF-1 $\alpha$ groups, rats received siRNAexpressing lentivirus; the rats in the TACE and control groups received lentivirus without siRNA. TACE was performed by placing a microcatheter into the gastroduodenal artery.

Results: The median survival time, body weight, and tumor volume of the siHIF- $1 \alpha+$ TACE group were better than those of the TACE, siHIF-1 $\alpha$, and control groups. A comparative analysis of the different treatment groups demonstrated that HIF-1 $\alpha$ RNAi could downregulate the levels of HIF-1 $\alpha$ and VEGF, inhibit tumor angiogenesis, and lessen metastases; all of these effects were enhanced by TACE.

Conclusion: HIF-1 $\alpha$ RNAi, which was administered in vivo in liver tumors under ultrasound guidance, improved the efficacy of TACE in treating hepatocellular carcinoma in an animal model.

Keywords: transarterial chemoembolization, hypoxia-inducible factor-1alpha, hepatocellular carcinoma, ultrasound guidance, RNA interference

\section{Introduction}

Transarterial chemoembolization (TACE) and transarterial embolization (TAE) are important options for patients with nonsurgical hepatocellular carcinoma (HCC) and have improved the overall survival rate. ${ }^{1,2}$ Both procedures are characterized by injecting embolic materials into the arteries supplying the tumor so as to induce ischemic necrosis. HCC cells themselves are hypoxic; embolization further increases hypoxia so the remaining tumor cells experience exacerbated hypoxia.

HIF-1 is a transcription factor consisting of HIF- $1 \alpha$ and HIF-1 $\beta$. Among them, HIF- $1 \alpha$ acts as a nuclear protein, which dictates the expression and activity of HIF-1 and transactivates genes. ${ }^{3}$ Under hypoxic conditions, prolyl hydroxylase domains are suppressed, and HIF- $1 \alpha$ acetylation is inhibited by histone deacetylase $1 .{ }^{4}$ Furthermore, HIF-1 $\alpha$ is stabilized, translocated to the nucleus, and heterodimerized with HIF-1 $\beta$ so as to form HIF-1. In the nucleus, HIF-1 induces the transcription of target genes that are implicated in events such as metabolism, cell survival/apoptosis, $\mathrm{pH}$ 
regulation, adhesion, extracellular matrix remodeling, angiogenesis, migration, and metastasis. ${ }^{5-7}$

Solid tumors tend to grow faster than the vasculature, therefore, rendering the tumor cells hypoxic. Tumor hypoxia increases HIF-1 $\alpha$ expression, which makes the tumor insensitive to chemotherapy and radiotherapy and increases tumor migration and invasion. ${ }^{8,9}$ Currently, substantial data indicate that HIF-1 is a crucial regulator of the cell response to hypoxia. ${ }^{10}$ Thus, HIF-1 $\alpha$ is considered as one of the preferred targets in therapy for solid tumors.

Several reports have shown that HIF-1 level increases in carcinomas following TACE or TAE. ${ }^{2,11,12}$ VEGF is a significant angiogenic factor. HIF-1 activates angiogenesis by the means of regulating the levels of VEGF and other related pro-angiogenic factors such as PDGF $\beta$ and PlGF. ${ }^{13}$ Shim et al reported that overexpression of VEGF related to poor prognosis following TACE in patients with HCC. ${ }^{14}$ Thus, if HIF-1 $\alpha$ was effectively inhibited, the efficacy of TACE and/or TAE in treating HCC might be improved.

In one study concerning RNA interference (RNAi) against HIF-1, researchers observed significantly decreased expression of VEGF and HIF-1 $\alpha$, and the induction of apoptosis in a hepatoma cell line as well. ${ }^{15}$ Another study demonstrated that TAE combined with intra-portal delivery of adeno-associated viral vectors expressing HIF-1 $\alpha$ antisense could improve the efficacy in treating HCC. ${ }^{16}$ Our previous study demonstrated that HIF- $1 \alpha$ RNAi reduced VEGF and HIF-1 $\alpha$ expression in vitro and improved the efficacy of TAE in vivo by decreasing the levels of VEGF and HIF-1 $\alpha$, suppressing tumor angiogenesis, inhibiting tumor growth, and attenuating metastasis. ${ }^{17}$ However, for the technology to be clinically applicable, HIF-1 $\alpha$ expression must be downregulated in vivo. ${ }^{17}$

As a strategy for cancer gene therapy, RNAi technology has attracted significant attention because of its robust gene silencing ability. ${ }^{18}$ RNAi involves the sequence-specific silencing of gene expression activated by synthesized small interfering RNAs (siRNAs), endogenous microRNAs, and other short double-stranded RNAs. ${ }^{19}$ RNAi has revolutionized our understanding of gene regulation.

In TAE, simple embolic agents are used to embolize the tumor-feeding arteries, whereas in TACE, a mixture of chemotherapeutic agents and embolic agents are injected into the tumor-feeding arteries. TACE has a broader clinical application than TAE; it is therefore more valuable to study TACE.

In this study, we investigated whether in vivo ultrasound (US)-guided RNAi against HIF-1 $\alpha$ enhanced the effects of TACE in treating HCC. The same viral vector, HIF-1 $\alpha$ RNAi sequence, cell line, animal model, and other characteristics described in our previous study were used for these experiments. ${ }^{17}$

\section{Materials and methods Animals}

Buffalo rats (Charles River Laboratories, Wilmington, MA, USA) were mated and kept in the Experimental Animal Research Center at Shanghai Medical School, Fudan University, People's Republic of China. Animals were maintained in cages and were allowed ad libitum access to standard water and rodent food. All animal studies were granted ethical approval by the Animal Research Committee of Zhongshan Hospital and were conducted in accordance with the animal care policy of Fudan University. Only male rats with a weight of $150 \mathrm{~g}$ to $200 \mathrm{~g}$ were chosen for the experiments.

\section{Cell lines}

The Buffalo rat hepatoma cell line McA-RH7777 was obtained from the American Type Culture Collection (no. CRL1601; ATCC, Manassas, VA, USA) and was maintained in Dulbecco's Modified Eagle's Medium supplemented with $1 \%$ penicillin and streptomycin (Thermo Fisher Scientific, Waltham, MA, USA) and 10\% fetal bovine serum (Thermo Fisher Scientific). The cell line was cultured at $37^{\circ} \mathrm{C}$ in a $95 \%$ humidified air, $5 \% \mathrm{CO}_{2}$ incubator. The culture medium was replaced every 3 days.

\section{Lentiviral expression vectors}

The two vectors for recombinant lentiviral expression utilized in the experiments were synthesized by GeneChem Corporation (Shanghai, People's Republic of China). One of them expressed HIF-1 $\alpha$-specific siRNA (sense strand: 5'-AACCAGTTGAATCTTCAGATA-3') as well as GFP, and the other vector, just expressing GFP (no siRNA), served as control.

\section{Animal model}

McA-RH7777 cells $\left(1 \times 10^{6} / 0.1 \mathrm{~mL}\right.$ phosphate-buffered saline) were subcutaneously injected as a cell suspension into the right thighs of the rats. The way to implant pieces of tumor tissue into the liver to establish rat model of HCC was the same as previously described. ${ }^{20}$

\section{US-guided in vivo transfection}

Tumor-bearing Buffalo rats were randomly divided into four groups $(n=10$ each): control, siHIF-1 $\alpha$, TACE, and siHIF-1 $\alpha+$ TACE. US-guided in vivo transfections were performed 2 weeks after tumor implantation, when the tumors 
were approximately $10 \mathrm{~mm}$ in diameter. The US equipment (ALOKA SSD-ALPHAS, Tokyo, Japan) was utilized in accordance with the manufacturer's guidelines. Animals were anesthetized via an intraperitoneal injection of trichloroacetaldehyde hydrate $(400 \mathrm{mg} / \mathrm{kg})$. The hair on the upper abdomen of the rats was removed, and the area was wiped with a medical coupling reagent (Aquasonic CLEAR, Parker Laboratories Inc., Fairfield, NJ, USA). A high frequency linear array probe (UST-5410; frequency: $15 \mathrm{~Hz}$; machine injury: 0.88) was chosen to directly image the upper abdomen (Figure 1). Using US imaging as a guideline, $4 \times 10^{8}$ transfection units of lentivirus with or without siRNA expression in $0.6 \mathrm{~mL}$ transduction reagent (GeneChem, Shanghai, People's Republic of China) was injected into the liver tumor using a standard $1 \mathrm{~mL}$ syringe (KDL, Kang Delai Corp., Shanghai, People's Republic of China). In the siHIF-1 $\alpha$ and siHIF- $1 \alpha+$ TACE groups, the rats were administered siRNAexpressing lentivirus; the rats in the TACE and control groups received lentivirus without siRNA. Lentivirus was injected into four locations in the tumors. Using US-guided imaging the four points were confirmed to provide sufficient distribution into the four corners of a 3D quadrilateral.

\section{TACE procedure}

TACE was performed 24 hours after US-guided transfection. The TACE procedure was performed as previously described. ${ }^{20}$ Briefly, the rats were anesthetized by intraperitoneally injecting trichloroacetaldehyde hydrate $(400 \mathrm{mg} / \mathrm{kg})$. A midline abdominal incision was performed to expose the gastroduodenal artery. After placing a Silastic tubing (SFM10350; SF Medical, Hudson, MA, USA) in the artery with the tip distal to the opening of the proper hepatic artery, a mixture of iodized oil (Lipiodol, Guerbet, Villepinte,

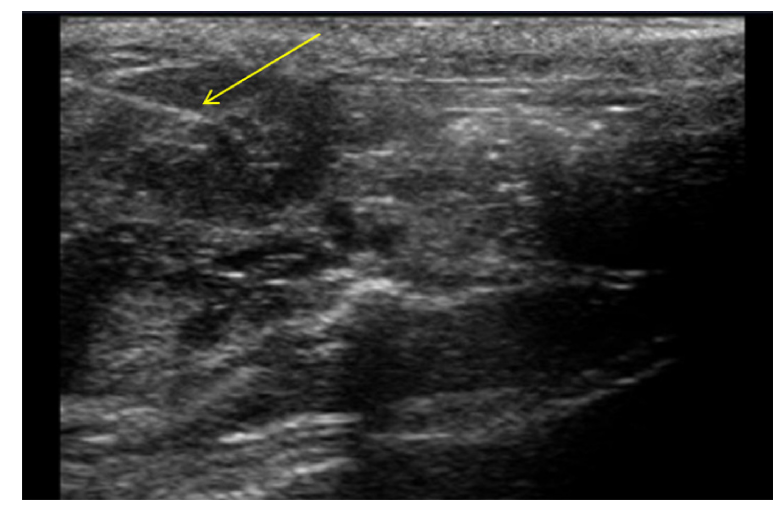

Figure I US-guided in vivo transfection in a rat.

Notes: Lentiviral vectors with or without siRNA were injected into a liver tumor using a I $\mathrm{mL}$ syringe (yellow arrow) under the guidance of US imaging. Abbreviations: US, ultrasound; siRNA, small interfering RNA.
France) and doxorubicin (Wanle Pharmaceutical Co., Ltd., Shenzhen, People's Republic of China) or just saline was slowly injected. Before TACE, the iodized oil was diluted 1:4 with saline, and then doxorubicin (final concentration: $0.125 \mathrm{mg} / \mathrm{mL}$ ) was added. In the TACE and siHIF-1 $\alpha+$ TACE groups, the animals were intra-arterially injected a mixture of iodized oil, doxorubicin, and saline (final volume: $1 \mathrm{~mL} / \mathrm{kg}$ ). In the control and siHIF- $1 \alpha$ groups, rats were administered the same amount of saline via the Silastic tubing (final volume: $1 \mathrm{~mL} / \mathrm{kg}$ ). A Nikon SMZ1500 dissection microscope (Nikon Corporation, Tokyo, Japan) was utilized in the operation of TACE.

\section{Body weight and tumor volume}

Body weights of the rats were recorded every week after intervention using an electronic scale. The tumor volume was measured by US imaging every week after treatment and was calibrated according to the following formula: $\left(\right.$ length $\times$ width $\left.^{2}\right) / 2 .^{21}$

\section{Rat survival and sample collection}

The date on which an animal died was recorded and the animals were dissected on the day they died. The lungs and liver were collected. Viable areas of the tumor were stored in liquid nitrogen for further examination of real-time reverse transcription polymerase chain reaction (RT-PCR), and the remaining areas were fixed in $10 \%$ formalin, embedded in paraffin, and sectioned $(3 \mu \mathrm{m})$.

\section{Immunohistochemistry}

The sections were stained for VEGF and HIF- $1 \alpha$ expression as well as microvessel density (MVD) utilizing the Catalyzed Signal Amplification System (Dako Denmark A/S, Glostrup, Denmark). After deparaffinization and rehydration of the slides, the process of antigen retrieval (Dako Denmark A/S) lasted for 45 minutes at $97^{\circ} \mathrm{C}$. Hematoxylin was used to counterstain the nuclei. Primary antibodies used in the experiments were as follows: mouse anti-CD31 monoclonal antibody 1:300 (Abcam, Hong Kong, People's Republic of China), mouse anti-VEGF $\alpha$ monoclonal antibody $(1: 1,500)$ (Abcam), and rabbit anti-HIF-1 $\alpha$ polyclonal antibody (1:500) (Sigma-Aldrich Co., St Louis, MO, USA).

For each slide, two or three fields were counted (400× magnification) to access the frequency of VEGF-positive cytoplasms and HIF-1 $\alpha$-positive nuclei. Twenty-two fields were counted in each group. VEGF and HIF-1 $\alpha$ expression were determined by the means of calculating the percentage of immunoreactive cells among the population of tumor cells. 
The MVD was determined to be the average microvessels in each field (400× magnification). ${ }^{22}$ Any area of obvious CD31-positive staining was considered as a single vessel. Twenty-four fields were checked in ten different tumors in every group. At least two of the authors, who were blinded to the identity of the sections, analyzed the section using a light microscope.

\section{Tumor metastasis and H\&E staining}

The left and right lung sections of the maximum coronal plane were treated by H\&E staining to assess the presence of metastasis. ${ }^{23}$ The number of lung nodules was counted under an optical microscope.

\section{Real-time RT-PCR}

After being harvested from tumor tissues using TRIzol (Thermo Fisher Scientific, Waltham, MA, USA), RNA underwent reverse transcription to cDNA utilizing a PrimeScript RT Reagent Kit (Takara Bio Inc., Shiga, Japan). Real-time PCR was conducted using a SYBR Premix Ex Taq Kit (Takara Bio Inc.) in accordance with the manufacturer's guidelines. Specific gene products from McA-RH7777 cell cDNA were amplified using the following primers: HIF-1 $\alpha$ antisense, 5'-TTG AAT CTG GGG GCA TGG TAA AAG-3'; HIF-1 $\alpha$ sense, $5^{\prime}$-TCC ATT ACC TGC CTC TGA AAC TCC-3'; VEGF $\alpha$ antisense, $5^{\prime}$-GCT GGC TTT GGT GAG GTT TGA T-3'; VEGF $\alpha$ sense, 5'-TCC AGG AGT ACC CCG ATG AGA TAG-3'; $\beta$-ACTIN antisense, 5'-GAG GCA TAC AGG GAC AAC ACA GC-3'; and $\beta$-ACTIN sense, $5^{\prime}$-CCT AAG GCC AAC CGT GAA AAG AT-3'. Comparative $\mathrm{Ct}$ method was adopted to calculate the relative gene expression levels: $2^{-\Delta \Delta \mathrm{Ct}}$, in which $\mathrm{Ct}$ represents the threshold cycle. ${ }^{24}$

\section{Statistical analysis}

The Statistical Package for the Social Sciences software for Windows, version 18.0 (SPSS Inc., Chicago, IL, USA) was used for statistical analyses. Continuous data are presented as mean \pm standard deviation. Among the four groups, VEGF and HIF-1 $\alpha$ expression, body weight, lung nodules, MVD, and tumor volume were compared by one-way analysis of variance and least significant difference (LSD) test. The overall survival was compared using the log-rank test. Differences in lung metastases were examined by Fisher's exact test. Differences were considered significant at $P<0.05$.

\section{Results}

All the rats successfully underwent the US-guided in vivo transfection and TACE procedures, the appetite and activity
Table I The number of rat deaths in each week (n)

\begin{tabular}{llllll}
\hline Group & \multicolumn{3}{l}{ Weeks after treatment } & Total \\
\cline { 2 - 5 } & $\mathbf{4}$ & $\mathbf{5}$ & $\mathbf{6}$ & $\mathbf{7}$ & \\
\hline siHIF-I $\alpha+$ TACE & 0 & 0 & 9 & $\mathrm{I}$ & 10 \\
siHIF-I $\alpha$ & 0 & 7 & 3 & 0 & 10 \\
TACE & 0 & 5 & 5 & 0 & 10 \\
Control & $\mathrm{I}$ & 9 & 0 & 0 & 10 \\
\hline
\end{tabular}

Abbreviation: TACE, transarterial chemoembolization.

level of the rats were mildly affected for 1-2 days following treatment, after which the appetite and activity level of the rats improved. The number of deaths in each group after treatment is presented in Table 1 by week. In the fourth week after treatment, a rat in the control group died. For statistical analysis, the tumor volume and body weight at death were considered as the data at the end of the fourth week after treatment. By the fifth week after treatment, all the rats of control group had died; therefore, the body weight and tumor volume were observed for 4 weeks after treatment.

\section{Overall survival}

The median survival of different treatment groups were as follows: siHIF-1 $\alpha+$ TACE group, 40 days (95\% confidence interval [CI]: 37.93-42.07 days); siHIF-1 $\alpha$ group, 33 days (95\% CI: 29.90-36.10 days); TACE group, 35 days (95\% CI: 31.90-38.10 days); and control group, 30 days (95\% CI: 28.99-31.01 days). The log-rank analysis of the survival for the four treatment groups is shown in Figure 2: the chi-square value was 35.82 , with a $P$-value of 0.001 . The siHIF-1 $\alpha+$ TACE

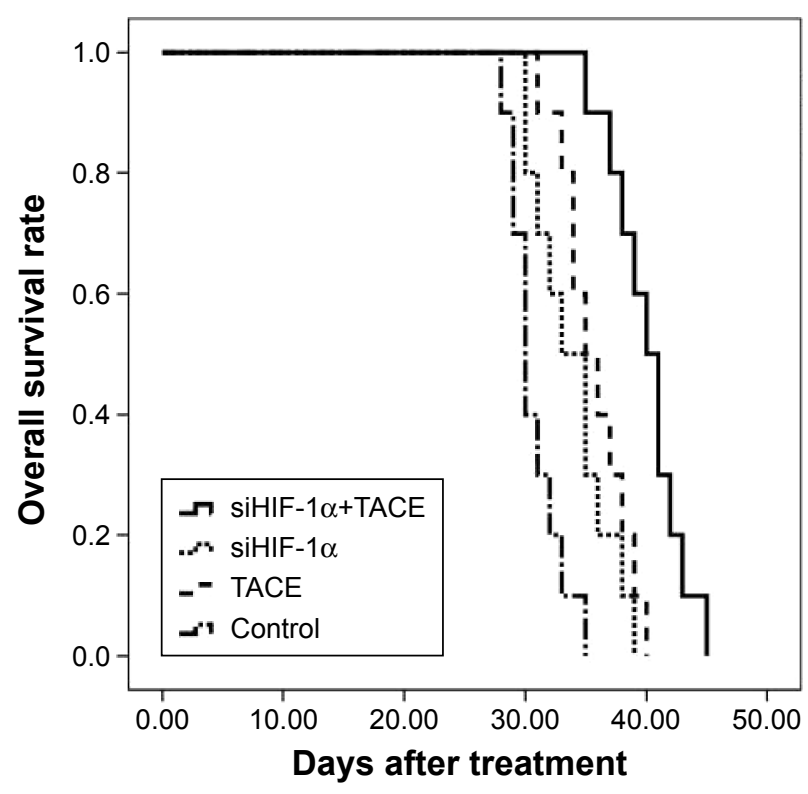

Figure 2 Log-rank survival analysis of the four treatment groups. Note: The chi-square value was 35.82 , and the $P$-value was 0.001 . Abbreviation: TACE, transarterial chemoembolization. 
group survived the longest, followed by the single treatment groups and then the control group. HIF-1 $\alpha$ RNAi improved the effectiveness of TACE in treating HCC by prolonging the rats' survival.

\section{Change in body weight}

The change in body weight of the rats in the four groups is shown in Figure 3. The body weight of the siHIF- $1 \alpha+$ TACE group continuously increased after treatment. However, the body weight in the other three groups declined after a brief increase. The mean body weights in the fourth week after treatment were as follows: siHIF- $1 \alpha+\mathrm{TACE}$ group, $242.80 \pm 10.93 \mathrm{~g} ;$ siHIF-1 $\alpha$ group, $195.00 \pm 13.22 \mathrm{~g}$; TACE group, $214.50 \pm 11.90 \mathrm{~g}$; and control group, $140.10 \pm 13.79 \mathrm{~g}$. The animals in the siHIF- $1 \alpha+$ TACE group were obviously heavier than those in the other three groups $(P=0.001$ for all comparisons); the animals in the siHIF- $1 \alpha$ and TACE groups were distinctly heavier than those in the control group ( $P=0.001$ for both comparisons). These results suggested that HIF-1 $\alpha$ RNAi enabled the animals to increase their body weight compared with the other treatment groups.

\section{Tumor volume}

The tumor volume measurements by means of US imaging were terminated 4 weeks after intervention. The tumor volume results are shown in Figure 4. Tumor growth in the siHIF$1 \alpha+$ TACE group was markedly inhibited, tumor growth in the TACE and siHIF-1 $\alpha$ groups was slightly inhibited, and tumor growth in the control group was obviously fast. The mean tumor volumes at the fourth week after treatment were as follows: siHIF-1 $\alpha+$ TACE group, $8,874 \pm 4,314 \mathrm{~mm}^{3}$; siHIF- $1 \alpha$

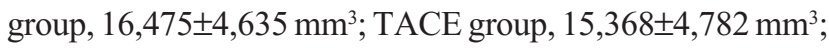
and control group, $21,350 \pm 4,588 \mathrm{~mm}^{3}$. At 4 weeks, tumor

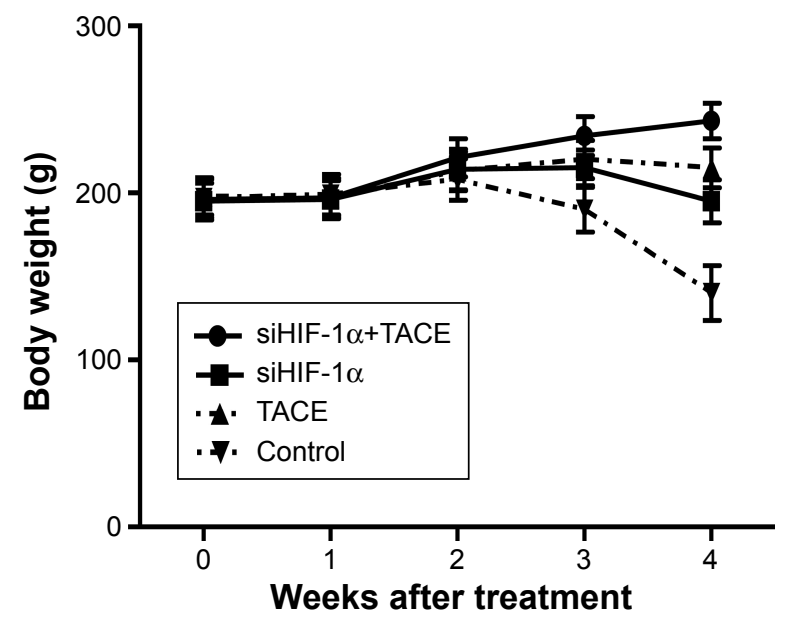

Figure 3 Body weight changes in the different groups after treatment. Abbreviation: TACE, transarterial chemoembolization.

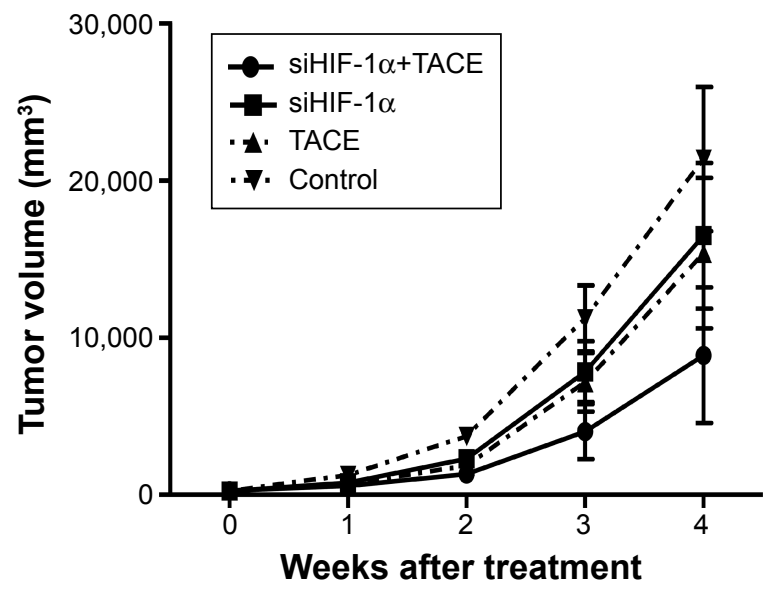

Figure 4 Tumor volume changes in the different groups after treatment as measured by US imaging.

Notes: Four weeks after treatment, the tumor volume was significantly smaller in the siHIF-I $\alpha+$ TACE group than in the other three groups ( $P=0.003$ vs siHIF- $\mid \alpha, P=0.001$ vs TACE, and $P=0.001$ vs control). The tumor volumes were significantly smaller in the siHIF-I $\alpha$ and TACE groups than in the control group ( $P=0.001$ for both comparisons). Abbreviations: US, ultrasound; TACE, transarterial chemoembolization.

volume was distinctly smaller in the siHIF- $1 \alpha+$ TACE group than those in the other three groups $(P=0.001$ vs siHIF- $1 \alpha$, $P=0.003$ vs TACE, and $P=0.001$ vs control); tumor volumes were obviously smaller in the siHIF- $1 \alpha$ and TACE groups than those in the control group $(P=0.023$ and 0.006 , respectively). These results suggested that HIF- $1 \alpha$ RNAi enhanced the effects of TACE in inhibiting HCC growth.

\section{Tumor metastasis}

Lung metastases lessened noticeably in response to siHIF- $1 \alpha$, from an incidence of $90 \%(9 / 10)$ in the control group to $40 \%$ $(4 / 10)$ in the siHIF- $1 \alpha$ group, and from $100 \%(10 / 10)$ in the TACE group to $50 \%(5 / 10)$ in the siHIF- $1 \alpha+$ TACE group (Figure 5A). The differences between the TACE-treated groups was statistically significant $(P=0.033)$. The average lung nodules were obviously less in the siHIF- $1 \alpha$ group than those in the control group ( $7.10 \pm 10.14$ vs $32.80 \pm 15.85$, $P=0.001)$ and were also less in the siHIF- $1 \alpha+$ TACE group than those in the TACE group ( $11.90 \pm 13.58$ vs $44.30 \pm 12.64$, $P=0.001$ ) (Figure 5B). HIF-1 $\alpha$ RNAi suppressed the lung metastases of HCC both individually and in conjunction with TACE.

\section{HIF-I $\alpha$,VEGF, and CD3 I expression}

Figure 6A-D shows the results of the immunohistochemical analysis of CD31, VEGF, and HIF-1 $\alpha$ expression. The average HIF-1 $\alpha$-positive staining in the siHIF- $1 \alpha+$ TACE, siHIF- $1 \alpha$, TACE, and control groups were 50.41 $\pm 13.28,44.67 \pm 11.86$, $70.26 \pm 14.22$, and $55.22 \pm 15.19$, respectively; the average VEGF-positive staining for the same groups were $58.64 \pm 14.03$, 
A

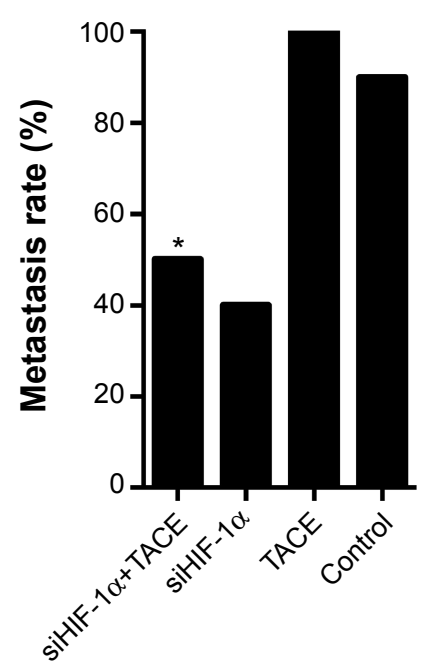

B

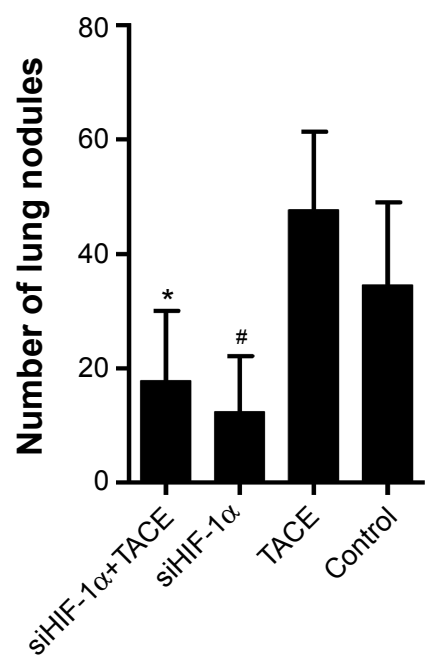

Figure 5 Comparison of the lung metastatic rate and number of lung nodules.

Notes: $(\mathbf{A})$ The lung metastasis rate was determined, and $(\mathbf{B})$ the number of lung nodules was counted. Significant differences $(P<0.05)$ compared with the TACE and control groups are denoted by * and ${ }^{\#}$, respectively.

Abbreviation: TACE, transarterial chemoembolization.

$53.37 \pm 12.28,80.53 \pm 15.10$, and $65.29 \pm 14.16$, respectively; and the average CD31-positive vessels per field $(400 \times)$ were $50.52 \pm 17.05,46.63 \pm 16.12,67.34 \pm 17.17$, and 56.29 \pm 14.81 , respectively. HIF-1 $\alpha$ knockdown significantly inhibited the tumor hypoxia-mediated increases in CD31, VEGF, and HIF-1 $\alpha$ expression $(P=0.043,0.007$, and 0.012 , respectively, compared with the control group). HIF-1 $\alpha$ knockdown also prevented the increase of CD31, VEGF, and HIF-1 $\alpha$ expression in response to TACE intervention and carcinoma hypoxia ( $P=0.001$ for all comparisons with the TACE group).

The results of RT-PCR analysis (Figure 7A, B) of HIF-1 $\alpha$ and VEGF expression were similar to the immunohistochemical analysis. The average relative mRNA expression levels of HIF-1 $\alpha$ were $5.26 \pm 1.49$, 4.38 $\pm 1.45,7.53 \pm 1.91$, and $6.04 \pm 1.84$ in the siHIF- $1 \alpha+$ TACE, siHIF- $1 \alpha$, TACE, and control groups, respectively. The mean relative mRNA expression levels of VEGF were 5.95 $\pm 1.44,5.25 \pm 1.50$, $8.41 \pm 1.65$, and $6.66 \pm 1.29$ in the siHIF- $1 \alpha+$ TACE, siHIF- $1 \alpha$, TACE, and control groups, respectively. HIF-1 $\alpha$ knockdown suppressed the tumor hypoxia-mediated increase in VEGF and HIF- $1 \alpha$ expression $(P=0.034$ and $P=0.040$, respectively, compared with control), and also prevented the increase of VEGF and HIF-1 $\alpha$ expression in response to TACE and tumor hypoxia ( $P=0.006$ and 0.002 , respectively, compared with the TACE group).

\section{Discussion}

Solid tumors tend to be hypoxic with up-regulated HIF-1 $\alpha$ expression, which leads to the following events: 1) VEGF is directly up-regulated, then, VEGF interacts with cell-surface receptors, VEGF receptor-1 and VEGF receptor-2, to regulate endothelial cell and tumor cell function to promote angiogenesis and alleviate tumor hypoxia; 2) pyruvate is converted into lactic acid in the cytoplasm, which increases glucose uptake, reduces oxygen metabolism, and places the tumor in a highly metabolic state; 3 ) the extracellular matrix is degraded by proteins such as E-cadherin and metalloproteases, which facilitates cancer cell migration and invasion. ${ }^{25-27}$ When the embolization treatment is performed, the degree of tumor hypoxia greatly increases. Many studies have demonstrated that HIF-1 $\alpha$ expression significantly increases in response to embolization. ${ }^{2,11,12}$

In our experiment, increased HIF-1 $\alpha$ expression in the control group merely resulted from tumor cell hypoxia, whereas the increased HIF- $1 \alpha$ expression in the TACE group represented both tumor cell hypoxia and embolizationmediated hypoxia. The reduction in HIF- $1 \alpha$ in the siHIF- $1 \alpha$ group in contrast to control suggested that the RNAi treatment inhibited the increased HIF- $1 \alpha$ expression caused by tumor hypoxia. The decrease in HIF- $1 \alpha$ expression in the siHIF-1 $\alpha+$ TACE group compared with the TACE group indicated that RNAi treatment inhibited the increased HIF-1 $\alpha$ expression in response to both tumor hypoxia and embolization.

Our study indicated that as HIF-1 $\alpha$ expression increased, VEGF expression and tumor migration increased. Although TACE can cause tumor volume to shrink by injecting chemotherapeutic agents and embolic materials directly into the arteries supplying the tumor, it further upregulated HIF-1 $\alpha$ 
A

siHIF- $1 \alpha+$ TACE

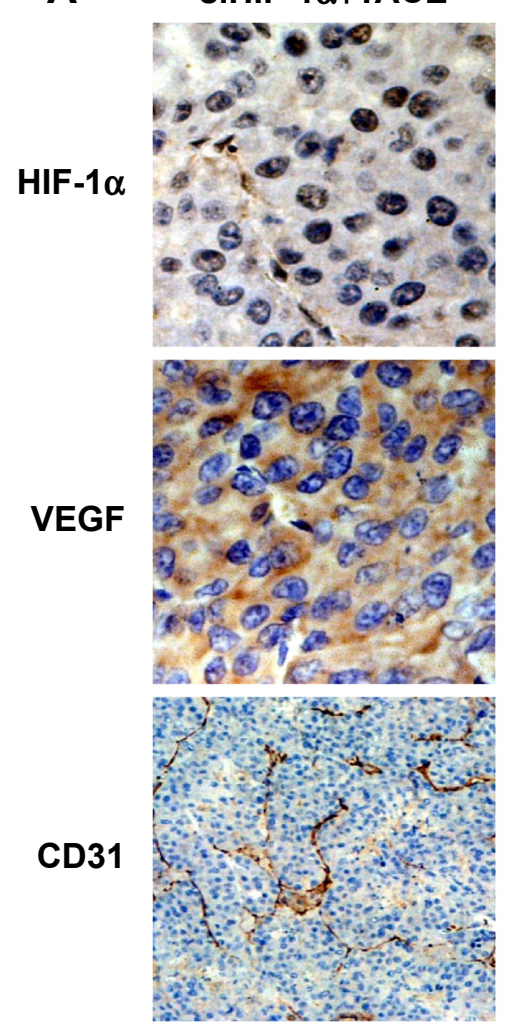

HIF-1 $\alpha$

B

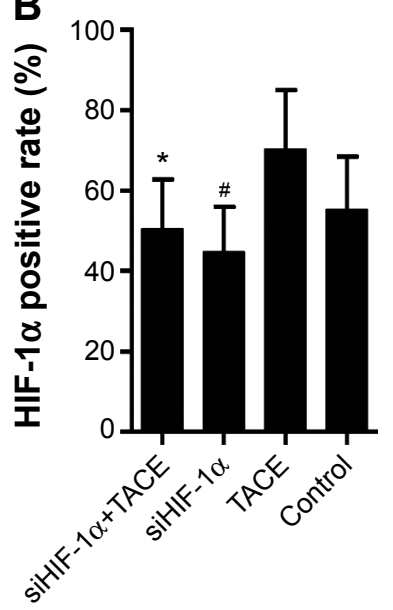

siHIF-1 $\alpha$
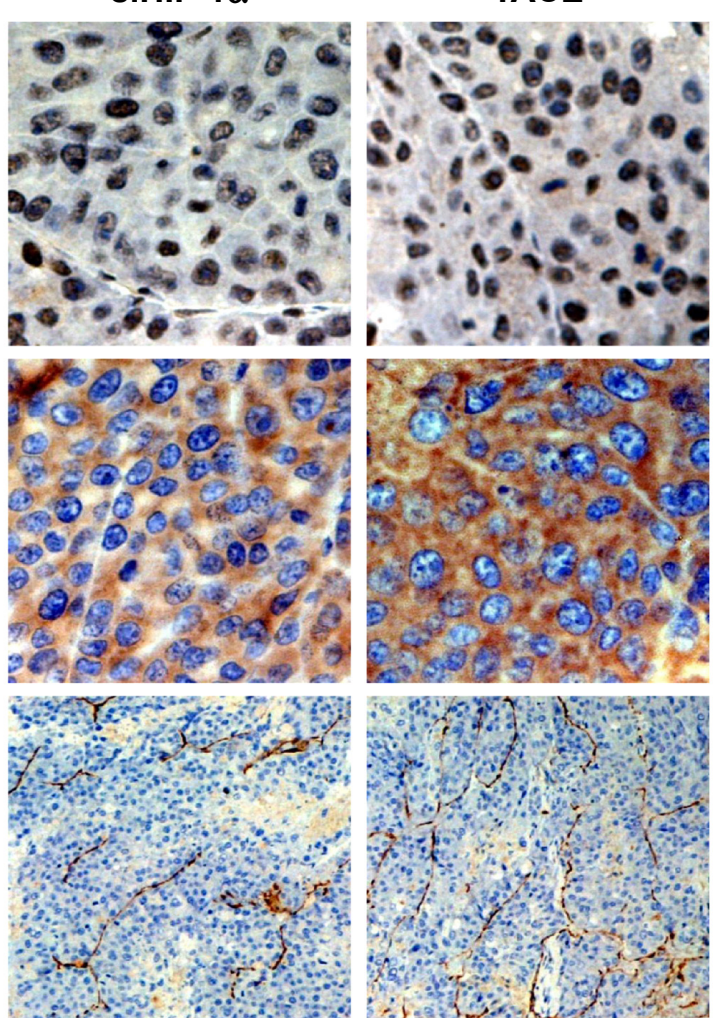

C

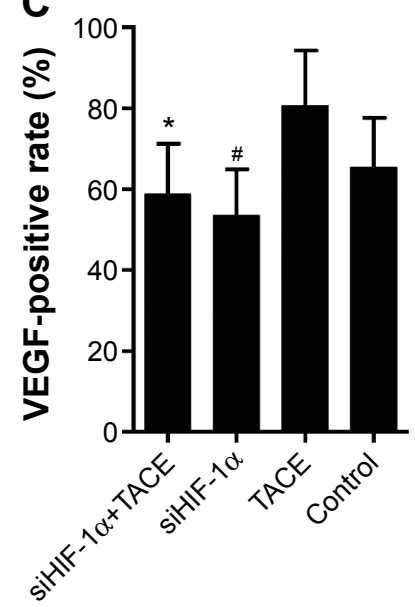

Control
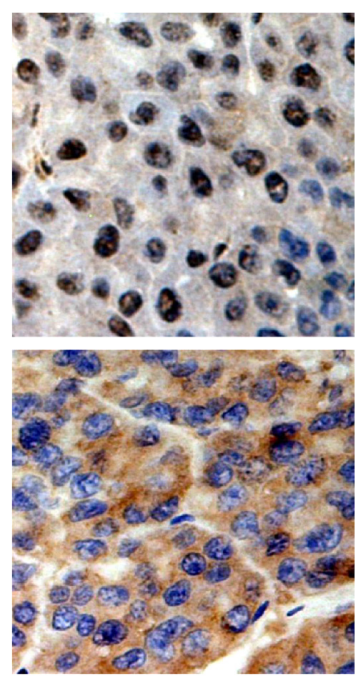

is
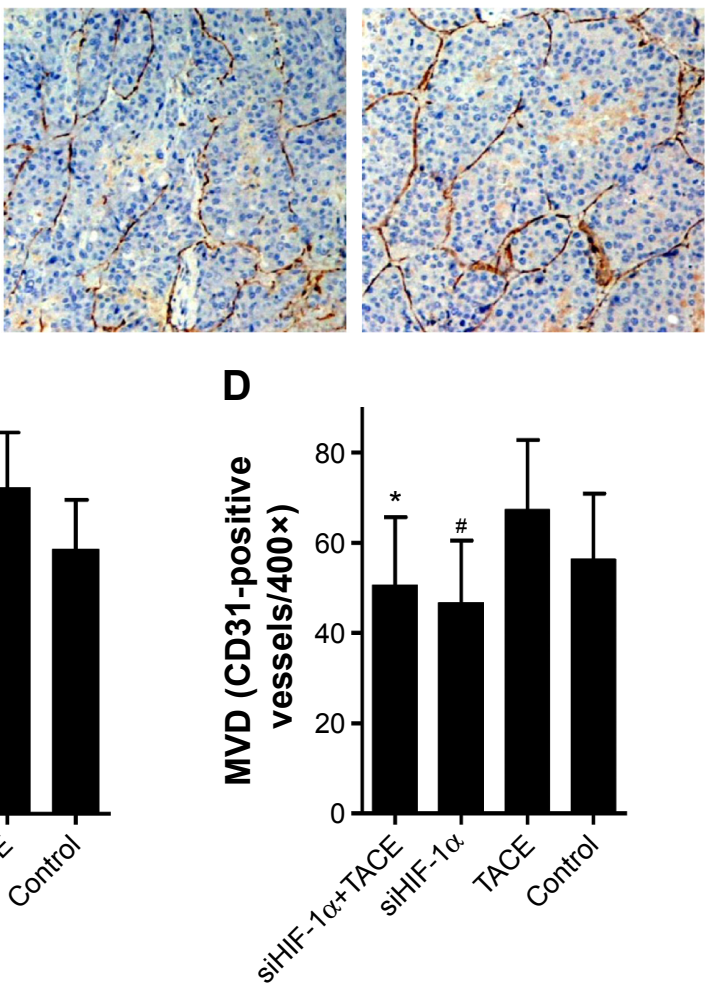

Figure 6 Immunohistochemical analysis.

Notes: (A-D) Immunohistochemical analysis of HIF-I $\alpha$, VEGF, and CD3I expression. Significant differences $(P<0.05)$ compared with the TACE and control groups are denoted by * and *, respectively.

Abbreviations: TACE, transarterial chemoembolization; MVD, microvessel density.

expression, thereby fostering increased VEGF expression and tumor migration. HIF-1 $\alpha$ RNAi suppressed the upregulation of HIF-1 $\alpha$ caused by tumor hypoxia alone or by both tumor hypoxia and embolization, reduced angiogenesis, and decreased tumor metastases. Therefore, HIF-1 $\alpha$ RNAi had the potential to improve the efficacy of TACE in treating HCC.

Tumor hypoxia elicits an increase in HIF-1 $\alpha$ expression, which can cause tumor resistance to chemotherapy and radiotherapy as well as reduce the efficiency of TACE in treating HCC. ${ }^{8,9}$ In our study, HIF-1 $\alpha$ expression was silenced by RNAi, resulting in the inhibition of VEGF expression, a prolonged survival time, a lack of body weight loss, and the reduction of metastases.

The results presented here correspond with those in our previous study. ${ }^{17}$ Both studies demonstrated that HIF-1 $\alpha$ RNAi improved the effects of TACE or TAE in treating HCC. However, this study more closely resembles clinical 
A

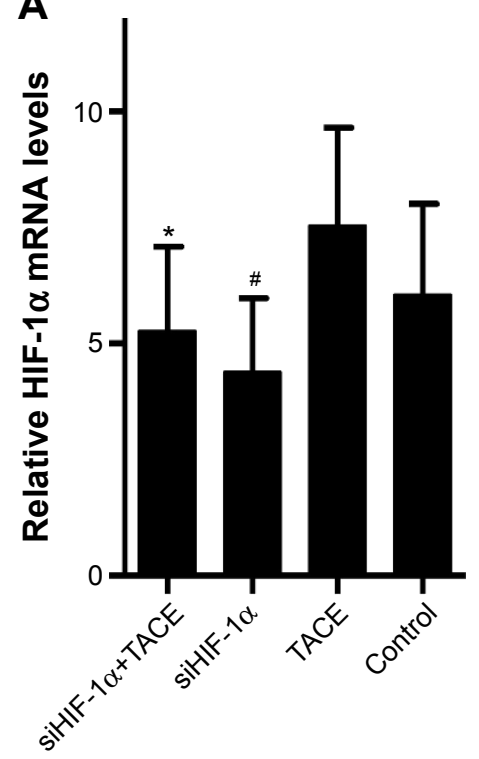

B

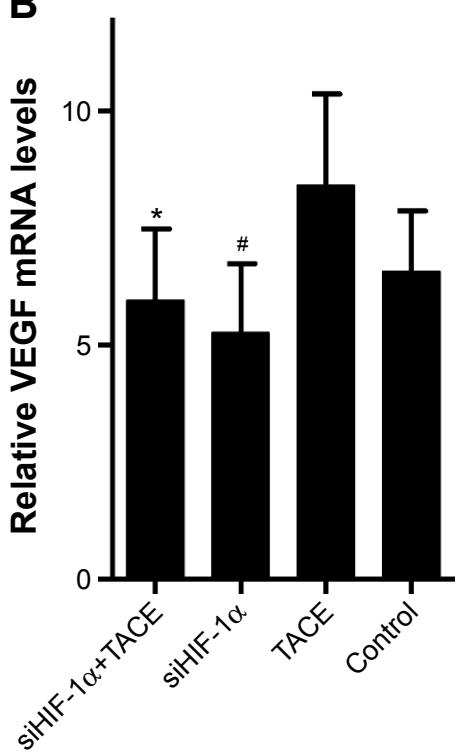

Figure 7 Real-time RT-PCR analysis.

Notes: (A, B) Real-time RT-PCR analysis of VEGF and HIF-I $\alpha$ expression. Significant differences $(P<0.05)$ compared with the TACE and control groups are denoted by * and ${ }^{*}$, respectively.

Abbreviations: TACE, transarterial chemoembolization; RT-PCR, reverse transcription polymerase chain reaction.

conditions than our previous study because we performed RNAi in vivo. ${ }^{17}$ This study also suggested the feasibility of intra-tumoral injections under imaging guidance as a method for delivering RNAi. The successful application of RNAi in the therapy of tumors depends heavily on the efficient delivery of specific siRNAs to the target lesions. When RNAi has been performed in vivo, subcutaneous tumor models have typically been used with intra-tumoral or intravenous siRNA delivery routes. ${ }^{28,29}$ US and an in situ liver tumor model were used in our study, and our study therefore more closely resembled clinical conditions.

The most widely used siRNAs are typically delivered via viral and nonviral methods. ${ }^{30}$ There are advantages and disadvantages to viral vectors and nonviral vectors. ${ }^{31}$ As the most effective gene delivery system, viral vectors have been applied in several clinical trials, but their potential side effects, intrinsic immunogenicity, and difficulties related to mass production limit their application in gene therapy. It is generally accepted that nonviral vector delivery systems are less efficient for in vivo gene transfer than viral vectors. However, they are less immunogenic, have a low risk of insertional mutagenesis, and quality control is generally easier than with viral vectors. Therefore, nonviral systems are an attractive tool for nucleic acid transfer. A viral vector was used in our study because of the importance of efficiency.
At present, RNAi has been recognized as a major area of research in medicine and biology, since it has great potential for RNAi-based therapeutics due to its high degree of specificity and efficacy. However, siRNA delivery to target cells has been a major hurdle. ${ }^{32}$ RNAi pathway can be activated by siRNA molecules after getting incorporated into the RNAi machinery. To diffuse across cell membranes and achieve target cell uptake, the large and hydrophilic siRNA molecules need the assistance of a delivery vector or a chemical modification. ${ }^{33}$ When administered systemically, siRNAs must overcome numerous physiological barriers to reach the target site, such as nonspecific interactions with serum proteins and nontarget cells, immune system, serum nucleases, renal clearance, and so on. ${ }^{34}$

Interventional radiology therapeutic techniques could be adopted for siRNA delivery to help overcome these physiological barriers. These image-guided techniques have the advantages of faster recoveries, fewer complications, and reduced cost in contrast to traditional therapies. ${ }^{35}$ Other studies have demonstrated that there was great potential for image-guided techniques to facilitate the delivery of RNAi molecules. ${ }^{36,37}$ Imaging guidance has been used for intra-arterial injections and intra-portal injections of RNAi molecules in vivo. ${ }^{38} \mathrm{We}$ utilized a US-guided local delivery technique to directly inject lentiviral vectors into tumors. The results suggested that image-guided delivery 
strategy might be feasible for RNAi-based gene delivery. This strategy avoided enzymatic hydrolysis by serum nucleases and nonspecific interactions with nontarget cells and proteins, prevented renal clearance, circumvented blood vessels to directly access the lesions, and reduced systemic toxicity.

\section{Conclusion}

In our study, US-guided delivery of HIF-1 $\alpha$ RNAi decreased VEGF and HIF- $1 \alpha$ expression in vivo, and HIF- $1 \alpha$ RNAi improved the effects of TACE in treating HCC in terms of preventing body weight loss, prolonging survival, inhibiting tumor angiogenesis and growth, and metastases. The use of US guidance may be advantageous in the clinical application of RNAi technology. Additional studies are necessary before image-guided techniques can be developed for clinical applications.

\section{Acknowledgments}

Grant support: The National Natural Science Foundation of China (Grant number 81171432); The Natural Science Foundation of Shanghai City (Grant number 15ZR1406700); The Health and Family Planning Commission of Shanghai City (Grant number 201440540).

\section{Disclosure}

The authors report no conflicts of interest in this work.

\section{References}

1. Llovet JM, Real MI, Montaña X, et al. Arterial embolisation or chemoembolisation versus symptomatic treatment in patients with unresectable hepatocellular carcinoma: a randomised controlled trial. Lancet. 2002;359(9319):1734-1739.

2. Rhee TK, Young JY, Larson AC, et al. Effect of transcatheter arterial embolization on levels of hypoxia-inducible factor-1alpha in rabbit VX2 liver tumors. J Vasc Interv Radiol. 2007;18(5):639-645.

3. Majmundar AJ, Wong WJ, Simon MC. Hypoxia-inducible factors and the response to hypoxic stress. Mol Cell. 2010;40(2):294-309.

4. Yoo YG, Kong G, Lee MO. Metastasis-associated protein 1 enhances stability of hypoxia-inducible factor-1alpha protein by recruiting histone deacetylase 1. EMBO J. 2006;25(6):1231-1241.

5. Brahimi-Horn MC, Chiche J, Pouysségur J. Hypoxia and cancer. J Mol Med (Berl). 2007;85(12):1301-1307.

6. Sendoel A, Kohler I, Fellmann C, Lowe SW, Hengartner MO. HIF-1 antagonizes p53-mediated apoptosis through a secreted neuronal tyrosinase. Nature. 2010;465(7298):577-583.

7. Pouyssegur J, Dayan F, Mazure NM. Hypoxia signalling in cancer and approaches to enforce tumour regression. Nature. 2006;441(7092): 437-443.

8. Semenza GL. Hypoxia-inducible factors in physiology and medicine. Cell. 2012;148(3):399-408.

9. Semenza GL. Hypoxia-inducible factors: mediators of cancer progression and targets for cancer therapy. Trends Pharmacol Sci. 2012;33(4): 207-214

10. Wang R, Zhou S, Li S. Cancer therapeutic agents targeting hypoxiainducible factor-1. Curr Med Chem. 2011;18(21):3168-3189.
11. Virmani S, Rhee TK, Ryu RK, et al. Comparison of hypoxia-inducible factor-1alpha expression before and after transcatheter arterial embolization in rabbit VX2 liver tumors. J Vasc Interv Radiol. 2008;19(10): 1483-1489.

12. Liang B, Zheng CS, Feng GS, et al. Correlation of hypoxia-inducible factor 1alpha with angiogenesis in liver tumors after transcatheter arterial embolization in an animal model. Cardiovasc Intervent Radiol. 2010; 33(4):806-812.

13. Kaseb AO, Hanbali A, Cotant M, Hassan MM, Wollner I, Philip PA. Vascular endothelial growth factor in the management of hepatocellular carcinoma: a review of literature. Cancer. 2009;115(21):4895-4906.

14. Shim JH, Park JW, Kim JH, et al. Association between increment of serum VEGF level and prognosis after transcatheter arterial chemoembolization in hepatocellular carcinoma patients. Cancer Sci. 2008; 99(10):2037-2044.

15. Mizuno T, Nagao M, Yamada Y, et al. Small interfering RNA expression vector targeting hypoxia-inducible factor 1 alpha inhibits tumor growth in hepatobiliary and pancreatic cancers. Cancer Gene Ther. 2006;13(2): $131-140$.

16. Sun $X$, Jiang $H$, Jiang $X$, et al. Antisense hypoxia-inducible factor-1alpha augments transcatheter arterial embolization in the treatment of hepatocellular carcinomas in rats. Hum Gene Ther. 2009;20(4):314-324.

17. Chen C, Wang J, Liu R, Qian S. RNA interference of hypoxia-inducible factor-1 alpha improves the effects of transcatheter arterial embolization in rat liver tumors. Tumour Biol. 2012;33(4):1095-1103.

18. Grimm D, Kay MA. RNAi and gene therapy: a mutual attraction. Hematology Am Soc Hematol Educ Program. 2007;473-481.

19. Hannon GJ. RNA interference. Nature. 2002;418(6894):244-251.

20. Kan Z, Kobayashi S, Phongkitkarun S, Charnsangavej C. Functional CT quantification of tumor perfusion after transhepatic arterial embolization in a rat model. Radiology. 2005;237(1):144-150.

21. Méndez O, Zavadil J, Esencay M, et al. Knock down of HIF-1alpha in glioma cells reduces migration in vitro and invasion in vivo and impairs their ability to form tumor spheres. Mol Cancer. 2010;9:133.

22. Kaira K, Oriuchi N, Takahashi T, et al. LAT1 expression is closely associated with hypoxic markers and $\mathrm{mTOR}$ in resected non-small cell lung cancer. Am J Transl Res. 2011;3(5):468-478.

23. Avwioro OG. Histochemistry and tissue pathology, principles and techniques. Nigeria: Claverianum press; 2010.

24. Livak KJ, Schmittgen TD. Analysis of relative gene expression data using real-time quantitative PCR and the 2(-Delta Delta C(T)) Method. Methods. 2001;25(4):402-408.

25. Wang XJ, Si LB. Advances on hypoxia inducible factor-1. Chin MedJ (Engl). 2013;126(18):3567-3571.

26. Wilson WR, Hay MP. Targeting hypoxia in cancer therapy. Nat Rev Cancer. 2011;11(6):393-410.

27. Meijer TW, Kaanders JH, Span PN, Bussink J. Targeting hypoxia, HIF-1, and tumor glucose metabolism to improve radiotherapy efficacy. Clin Cancer Res. 2012;18(20):5585-5594.

28. Yamaguchi K, Feril LB Jr, Tachibana K, et al. Ultrasound-mediated interferonbeta gene transfection inhibits growth of malignant melanoma. Biochem Biophys Res Commun. 2011;411(1):137-142.

29. Carson AR, McTiernan CF, Lavery L, et al. Ultrasound-targeted microbubble destruction to deliver siRNA cancer therapy. Cancer Res. 2012;72(23):6191-6199.

30. Chen J, Xie J. Progress on RNAi-based molecular medicines. Int J Nanomedicine. 2012;7:3971-3980.

31. Gavrilov K, Saltzman WM. Therapeutic siRNA: principles, challenges, and strategies. Yale J Biol Med. 2012;85(2):187-200.

32. Nikitenko NA, Prassolov VS. Non-Viral Delivery and Therapeutic Application of Small Interfering RNAs. Acta Naturae. 2013;5(3):35-53.

33. Whitehead KA, Langer R, Anderson DG. Knocking down barriers: advances in siRNA delivery. Nat Rev Drug Discov. 2009;8(2): $129-138$.

34. Kanasty R, Dorkin JR, Vegas A, Anderson D. Delivery materials for siRNA therapeutics. Nat Mater. 2013;12(11):967-977. 
35. Solomon SB, Silverman SG. Imaging in interventional oncology. Radiology. 2010;257(3):624-640.

36. Xing R, Liu G, Zhu J, Hou Y, Chen X. Functional Magnetic Nanoparticles for Non-Viral Gene Delivery and MR Imaging. Pharm Res. 2014;31(6):1377-1389.
37. Panje CM, Wang DS, Willmann JK. Ultrasound and microbubblemediated gene delivery in cancer: progress and perspectives. Invest Radiol. 2013;48(11):755-769.

38. Duan F, Lam MG. Delivery approaches of gene therapy in hepatocellular carcinoma. Anticancer Res. 2013;33(11):4711-4718.

\section{Publish your work in this journal}

OncoTargets and Therapy is an international, peer-reviewed, open access journal focusing on the pathological basis of all cancers, potential targets for therapy and treatment protocols employed to improve the management of cancer patients. The journal also focuses on the impact of management programs and new therapeutic agents and protocols on

\section{Dovepress}

patient perspectives such as quality of life, adherence and satisfaction. The manuscript management system is completely online and includes a very quick and fair peer-review system, which is all easy to use. Visit http://www.dovepress.com/testimonials.php to read real quotes from published authors. 